\title{
Cortical Folding Analysis on Patients with Alzheimer's Disease and Mild Cognitive Impairment
}

\author{
David M. Cash ${ }^{1,2}$, Andrew Melbourne ${ }^{1}$, Marc Modat ${ }^{1}$, M. Jorge Cardoso ${ }^{1}$, \\ Matthew J. Clarkson ${ }^{1}$, Nick C. Fox ${ }^{2}$, and Sebastien Ourselin ${ }^{1,2}$ \\ ${ }^{1}$ Centre for Medical Image Computing, University College of London, UCL ${ }^{\star}$ \\ ${ }^{2}$ Dementia Research Centre, University College of London, UCL
}

\begin{abstract}
Cortical thinning is a widely used and powerful biomarker for measuring disease progression in Alzheimer's disease (AD). However, there has been little work on the effect of atrophy on the cortical folding patterns. In this study, we examined whether the cortical folding could be used as a biomarker of AD. Cortical folding metrics were computed on 678 patients from the Alzheimer's Disease Neuroimaging Initiative (ADNI) cohort. For each subject, the boundary between grey matter and white matter was extracted using a level set technique. At each point on the boundary two metrics characterising folding, curvedness and shape index, were generated. Joint histograms using these metrics were calculated for five regions of interest (ROIs): frontal, temporal, occipital, and parietal lobes as well as the cingulum. Pixelwise statistical maps were generated from the joint histograms using permutations tests. In each ROI, a significant reduction was observed between controls and AD in areas associated with the sulcal folds, suggesting a sulcal opening associated with neurodegeneration. When comparing to MCI patients, the regions of significance were smaller but overlapping with those regions found comparing controls to AD. It indicates that the differences in cortical folding are progressive and can be detected before formal diagnosis of AD. Our preliminary analysis showed a viable signal in the cortical folding patterns for Alzheimer's disease that should be explored further.
\end{abstract}

\section{Introduction}

Alzheimer's disease (AD) is a progressive neurodegenerative disorder that affects nearly 1 in 8 people over 65 in the U.S. [1, and the prevalence is expected to increase in the near future. The hallmark pathology of AD are amyloid plaques and neurofibrillary tangles, which result in neuronal dysfunction. This ultimately leads to cell death which can be observed macroscopically in structural MRI as brain atrophy. Numerous imaging biomarkers have been proposed for the robust

\footnotetext{
* We would like to acknowledge UK registered charity SPARKS, the National Institute for Health Research (NIHR), the Fundação para a Ciência e a Tecnologia, Portugal, the EPSRC (EP/H046410/1), and the CBRC Strategic Investment Award (Ref. 168).
} 
and accurate measurement of this atrophy process 223. One of the most popular methods has been to measure cortical thickness 445. In this method, the cortical ribbon is extracted and correspondences are established between the grey matter (GM) to white matter (WM) boundary and the pial surface, from which thickness measurements can then be computed. Cortical thickness is a biomarker that has been shown to separate patients with $\mathrm{AD}$ from healthy controls [5], and to find a pattern of thinning [6] that closely resembles what is observed through postmortem studies [7]. In [8], there were some regions, primarily in the temporal lobe that appeared to thin at greater rates if the subject showed signs of Alzheimer's disease pathology according to cerebrospinal fluid markers of amyloid beta and tau.

Besides cortical thickness, the cortical folding pattern has been analysed for numerous applications. Manual histology based methods frequently use a gyrification index (GI) defined on slices measuring the ratio of the length of the grey matter to that of the shortest route around the coronal surface [910]. Computational methods using structural MRI require an accurate underlying segmentation and are in principle defined in 3D. 11] made use of a level set routine in order to assess the scaling relation of cortical volume to cortical surface area and developed summary statistics of the cortical surface by collecting complementary values of curvature into two-dimensional histograms. The representation of the surface is such that their histograms have two visible peaks distinguishing gyri from sulci. The histograms can be assessed using pixelwise statistical tests. In 12] the authors analysed the cortical surface by defining a mesh on the surface of the cortex and defining a mean curvature found from nodes within a local region. This study of healthy adults found strong gender dimorphism although the impact of overall subject size was unclear. The longitudinal cortical folding pattern of neonates has also been investigated by [13] in which after a semiautomatic segmentation, the cortical surface was delineated by a surface mesh. The authors analysed the appearance and formation of major sulci over 26-36 weeks gestational age making use of a sulcation ratio of the area of major sulci in relation to total cortical area.

Despite the volume of work on cortical folding, there has been very little focus on examining how it is affected by the process of neurodegeneration. King et al. 14. used fractal dimension analysis and gyrification index in addition to cortical thickness measurements to study the cortical ribbon in patients with AD. In this study, we applied the curvature metrics defined in [11] to determine if there are group wise differences in cortical folding patterns between normal controls (NC), mild cognitively impaired (MCI), and patients with AD.

\section{Methods}

\subsection{Data}

The data for this study consisted of a total of 678 participants, divided into 4 groups: controls ( $\mathrm{n}=199$, age 76.0 (5.1), 47\% female, MMSE 29.1 (1.0)), MCI patients stable after followup of up to 36 months $(n=192$, age $75.2(7.1), 34 \%$ 
female, MMSE 27.0 (1.8)), MCI patients who converted to AD during followup $(\mathrm{n}=140$, age $74.3(7.4), 39 \%$ female, MMSE $27.1(1.7))$ and 147 subjects diagnosed with AD (mean age 75.3 (7.3), 47\% female, MMSE 23.4 (1.9)). All subjects were selected from the Alzheimer's Disease Neuroimaging Initiative (ADNI) database [15] A $1.5 \mathrm{~T}$ scan with sufficient image quality from each subject's baseline visit was downloaded from the ADNI database. All images went through the standard pre-processing pipeline of bias correction, correction of $B_{1}$ inhomogeneity, and correction of geometric distortion [16]. The whole brain was manually delineated through a semi-automated process [17], which was used for further calculations.

\subsection{Tissue Classification and Brain Parcellation}

The pre-processed images were masked using the brain delineations and then segmented using an expectation-maximisation based algorithm [18] into five tissue classes: cortical GM, WM, external CSF, deep GM and internal CSF. The image was also parcellated into anatomical regions of interest. A library of 30 atlases manually labelled with 83 anatomical regions [19] was used as a basis for the parcellations. Each of the atlases was propagated via a nonlinear registration to provide a set of candidate segmentations for the new image. These candidate parcellations were combined using a label fusion strategy based on the STAPLE method [20]. It was adapted to use only a subset of the most similar candidate segmentations chosen for each voxel by a local image similarity metric [21].

\subsection{Boundary Surface Generation}

The segmentation method allows an inspection of the shape of the boundary between grey and white matter surfaces similar to [1]. In our work we optimise the boundary between GM and WM using an evolving level set, solving a partial differential equation (11) for the function $\phi(\mathbf{x}, t)$, consisting of a weighted signed distance function from the boundary, $d$, and a curvature function, $c$, that serves as a regularisation term. The level set is initialised at $p(\mathrm{WM})=0.5$, that provides a signed distance function from the GM/WM boundary.

$$
\frac{\delta \phi}{\delta t}+\left(\lambda_{d} d(\mathbf{x}, t)-\lambda_{c} c(\mathbf{x}, t)\right)\|\nabla \phi(\mathbf{x}, t)\|=0
$$

The outer boundary of GM can be defined by evolving the initial GM/WM surface outward to the $p(\mathrm{WM})+p(\mathrm{GM})=0.5$ boundary. The ratio of the inner surface (GM/WM interface) area to the outer surface area (GM/CSF interface) can be used as an approximation of the gyrification index [9].

\subsection{Curvature Measurements}

We define measures of curvature on this implicit surface, similar to the method used in [1]. The local Hessian matrix of second order derivatives can be found

\footnotetext{
1 WWW.adni-info.org
} 
at each point of this implicit surface. Hence, at every point on the surface we can use the eigenvalues of the local Hessian matrix, $\kappa_{1}$ and $\kappa_{2}$ (with $\kappa_{1}>\kappa_{2}$ ) to summarise the local shape. Explicitly we define the shape index, $S$ (2), describing how cup-like or saddle-like the surface is,

$$
S=\frac{2}{\pi} \tan ^{-1}\left(\frac{\kappa_{2}+\kappa_{1}}{\kappa_{2}-\kappa_{1}}\right)
$$

and a curvature, $C$, describing the distortion of the surface relative to that of a flat sheet $\left([22): C=\sqrt{\left(\kappa_{1}\right)^{2}+\left(\kappa_{2}\right)^{2}}\right.$. Intuitively $C$ and $S$ are complementary in the sense that they are a polar representation of the eigenvalue sum $\left(\kappa_{1}+\kappa_{2}\right)$ and difference $\left(\kappa_{2}-\kappa_{1}\right)$. The value of $C$ is corrected for brain volume using the strategy described in [11, where each value of $C$ is divided by a correction factor: $\beta_{i}^{3}=\overline{I C V} / I C V_{i}$, where ICV is the intracranial volume.

The distribution of $C$ and $S$ can be summarised in a 2D histogram [11, in which empirically, the resulting histograms have two clusters corresponding to negative (sulci) and positive shape index (gyri). Joint histograms were constructed from these pairs of values at every point on the level set. We used a joint histogram of 32 bins along each axis, with a range for curvature from 0 to 1.5 and shape index from -1 to 1 . In order to provide more spatial localisation to the measurements, the joint histograms were built over various regions of interest (ROI) as defined by the parcellation. Individual regions were combined into 5 meta regions: the cingulum, frontal, occipital, parietal, and temporal lobes. From the joint histogram, a bivariate probability density function (PDF) was estimated using an adaptive kernel density estimation technique 23] based on the discrete cosine transform.

\subsection{Statistical Analysis}

Pixelwise statistical tests were performed on the joint histogram for each ROI's from all of the subjects. The resulting t-statistic values were corrected for multiple comparisons using a non-parameteric method 24. 5000 permutations were performed on 12 t-tests for each region. These t-tests represent forward (i.e. control $>\mathrm{AD}$ ) and reverse (i.e. AD > control) comparisons between each of the four groups. Age and gender were accounted for in the design matrix as covariates.

\section{Results}

Mean GI values, adjusted for TIV, gender and age, for the four groups are shown in Fig. 1. There were significant differences for controls vs. MCI-Stable $(p=0.027)$, controls vs. MCI-Converters $(p=0.011)$, controls vs. AD $(p<$ $0.001)$, and MCI Stable vs AD $(p=0.039)$. There was no difference between the two MCI subgroups $(p=0.617)$ or MCI Converters vs. AD $(p=0.147)$.

Figure 2 shows the average histogram for each ROI overlaid with colour coded areas when a t-test reached significance at $(p<0.05)$ after correcting for multiple comparisons. In all five ROIs, there are regions of statistical significance (red, top 


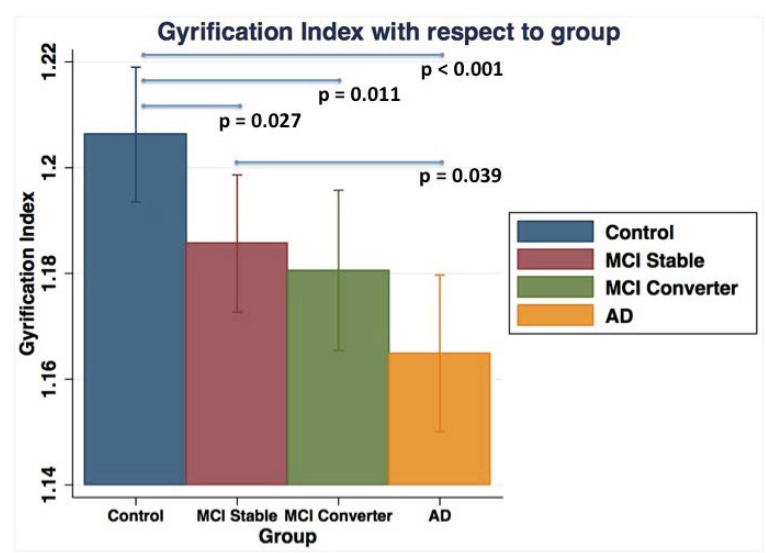

Fig. 1. Mean (95\% confidence intervals) of gyrification index for each of the groups. The values have been adjusted for age, gender and total intracranial volume.

row) that indicate where the values in the histogram are higher in controls than $\mathrm{AD}$. These occur mostly in areas near the peak of the joint histogram, where $\mathrm{S}$ is negative and $\mathrm{C} \approx 0.2-0.5 \mathrm{~mm}^{-1}$. For the cingulum, there is also a region at a similar curvature but where $\mathrm{S}$ is positive. Comparisons involving MCI subgroups resulted in smaller regions primarily contained within the control $>\mathrm{AD}$ region. Significant regions were detected for control $>$ MCI Converters (all ROIs except frontal), controls > MCI Stable (frontal, temporal, parietal), MCI Stable > AD (temporal, parietal), and MCI Converter > AD (temporal).

In all five ROIs, there were areas of significance (red regions, bottom row) when using the reverse comparison, $\mathrm{AD}>$ Control. For each ROI except the cingulum, these occurred in two areas of the histogram: (1) upper left, where $\mathrm{S}$ is negative and $\mathrm{C}>0.65 \mathrm{~mm}^{-1}$ and (2) far right, where $\mathrm{S}$ is close to +1 and $\mathrm{C} \approx 0.2 \mathrm{~mm}^{-1}$. In the cingulum, there were also significant regions in the very lower left: where $\mathrm{S}$ is negative and $\mathrm{C}<0.175 \mathrm{~mm}^{-1}$. Similar smaller overlapping regions were again observed when performing comparisons with MCI subgroups including: MCI Converter > Control (temporal, occipital), MCI Stable > Control (cingulum), AD > MCI Stable (frontal,temporal parietal).

\section{Discussion}

The cortical folding measurements in this paper track changes between disease groups that appear to be related to neurodegeneration. In Fig. 1, there are statistically significant differences between a summary statistic derived from the cortical folding. This value is higher in controls than MCI and AD subjects, which was also observed by King et al. [14, indicating the complexity of cortical folding is decreasing due to the disease process.

The main areas of significance in the forward comparison are near one of the histogram peaks where $S$ is negative and $C$ is moderate, typically attributed 


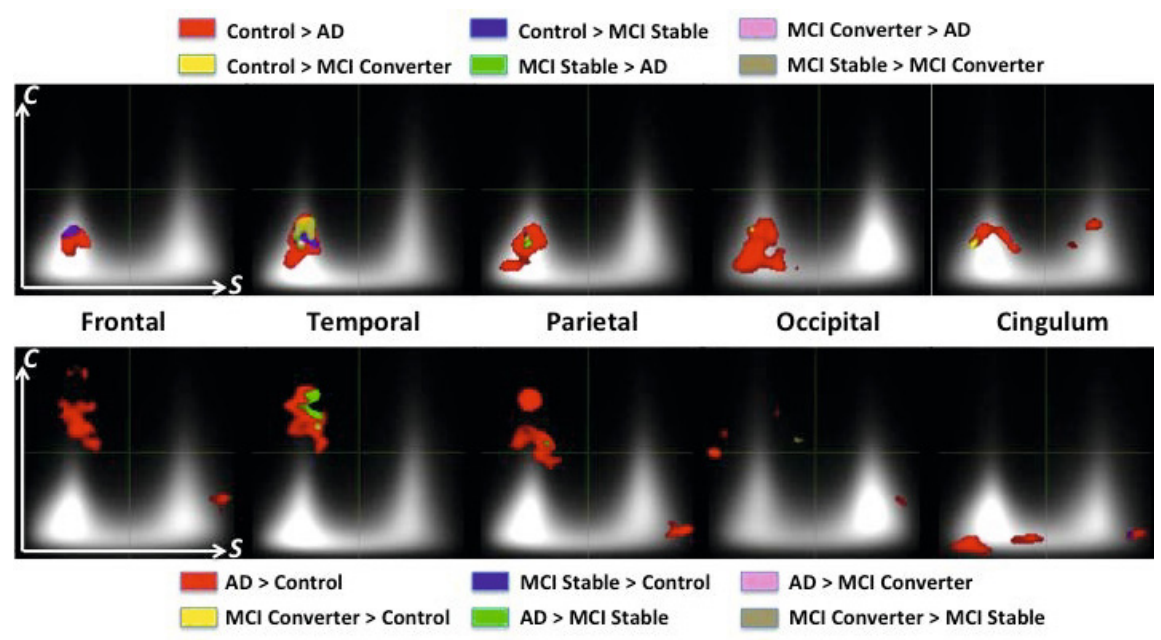

Fig. 2. Statistical maps for each region of interest. The $\mathrm{X}$ axis represents the shape index (range -1 to +1 , unitless) and the $\mathrm{Y}$ axis is the curvature value (range 0 to $1.5 \mathrm{~mm}^{-1}$ ). The top row has the forward comparisons (i.e. control $>\mathrm{AD}$ ) and the bottom row the reverse comparisons (i.e. $\mathrm{AD}>$ control). Areas of statistical significance $(p<0.05)$ are overlaid on the average joint histogram as colour coded in the legend.

to sulcal areas. As these regions are greater in healthier patients (i.e Control $>$ MCI > AD), this suggests that the sulci are widening and thus the curvature is reducing, thus flattening the peak of the histogram. There are also regions of significance when performing reverse comparisons. These occur both in extremely high curvature areas with negative shape indices or extremely low curvature areas with positive shape indices (and also in negative shape indices in the case of the cingulum). The high curvature areas have much lower values $(5-30 \%)$ in the PDF compared to the areas representing the sulcal peaks. It could either be that at this image resolution, the level set is unable to accurately acquire these areas of high curvature in healthy controls until there is further sulcal opening, or that neurodegeneration is further opening deep sulcal spaces and increasing cortical folding complexity. The low curvature areas being higher in affected patients seems to support the idea that neurodegeneration is reducing the curvature in the cortical folds. It is encouraging that the comparisons involving MCI subgroups result in smaller clusters that overlap with regions associated with controls vs. AD. This appears to suggest the measurement tracks with disease progression. The temporal lobe appears to show the greatest discriminant power, as it showed the most discrimination between the four groups. This is in agreement with many studies which indicate structures in the temporal lobe are some of the earliest to change.

It will be important to compare this method with cortical thickness measurements to determine if there is a correlation between the two measurements. As there is significant cortical variability between subjects, it would be difficult to apply this method on a vertex or voxel wide basis as is done in cortical thickness. 
However, it would be worthwhile to compute the joint histograms over smaller ROIs defined, such as individual cortical regions from the parcellation. Often in clinical trials, a summary statistic is more desirable than statistical maps to asses the effectiveness of treatment. Therefore, it is imperative that effective summary statistics, similar to the gyrification index, are developed from these metrics. As the level set can be evolved outwards to the pial surface, it would be worthwhile to test the effect of computing the curvature metrics from different boundaries, such as the outer pial surface or a midline between the two boundaries, as King found different levels of correlation between their fractal dimension values and the cortical thickness depending on which boundary was used.

\section{Conclusion}

From this preliminary study, there is evidence that the cortical folding pattern could be a useful biomarker in disease progression of Alzheimer's disease. While primarily applied to areas of development (such as neonatal applications), these markers also apply to neurodegeneration as well. Further work to refine the method and determine longitudinal changes should be pursued.

\section{References}

1. Alzheimer's Association, Alzheimer's facts and figures

2. Holland, D., Dale, A.M.: Nonlinear registration of longitudinal images and measurement of change in regions of interest. Med. Image Anal. 15(4), 489-497 (2011)

3. Leung, K.K., Ridgway, G.R., Ourselin, S., Fox, N.C.: Consistent multi-time-point brain atrophy estimation from the boundary shift integral. NeuroImage 59(4), 3995-4005 (2011)

4. Fischl, B., Dale, A.M.: Measuring the thickness of the human cerebral cortex from magnetic resonance images. PNAS 97(20), 11050-11055 (2000)

5. Acosta, O., Bourgeat, P., Zuluaga, M., Fripp, J., Salvado, O., Ourselin, S.: Automated voxel-based 3D cortical thickness measurement in a combined Lagrangian-Eulerian PDE approach using partial volume maps. Med. Image Anal. 13(5), 730-743 (2009)

6. Dickerson, B.C., Bakkour, A., Salat, D.H., Feczko, E., Pacheco, J., Greve, D.N., Grodstein, F., Wright, C.I., Blacker, D., Rosas, H.D., Sperling, R.A., Atri, A., Growdon, J.H., Hyman, B.T., Morris, J.C., Fischl, B., Buckner, R.L.: The cortical signature of Alzheimer's disease: regionally specific cortical thinning relates to symptom severity in very mild to mild AD dementia and is detectable in asymptomatic amyloid-positive individuals. Cerebral Cortex 19(3), 497-510 (2009)

7. Braak, H., Braak, E.: Neuropathological stageing of Alzheimer-related changes. Acta Neuropathologica 82(4), 239-259 (1991)

8. Desikan, R.S., Sabuncu, M.R., Schmansky, N.J., Reuter, M., Cabral, H.J., Hess, C.P., Weiner, M.W., Biffi, A., Anderson, C.D., Rosand, J., Salat, D.H., Kemper, T.L., Dale, A.M., Sperling, R.A., Fischl, B.: Selective disruption of the cerebral neocortex in Alzheimer's disease. PloS One 5(9), e12853 (2010)

9. Zilles, K., Armstrong, E., Schleicher, A., Kretschmann, H.J.: The human pattern of gyrification in the cerebral cortex. Anat. Embryol. 179, 173-179 (1988) 
10. Armstrong, E., Schleicher, A., Omran, H., Curtis, M., Zilles, K.: The ontogeny of human gyrification. Cerebral Cortex 5(1), 56-63 (1995)

11. Awate, S.P., Yushkevich, P.A., Song, Z., Licht, D.J., Gee, J.C.: Cerebral cortical folding analysis with multivariate modeling and testing: Studies on gender differences and neonatal development. NeuroImage 53(2), 450-459 (2010)

12. Luders, E., Thompson, P.M., Narr, K.L., Toga, A.W., Jancke, L., Gaser, C.: A curvature-based approach to estimate local gyrification on the cortical surface. NeuroImage 29(4), 1224-1230 (2006)

13. Dubois, J., Benders, M., Borradori-Tolsa, C., Cachia, A., Lazeyras, F., Ha-Vinh Leuchter, R., Sizonenko, S.V., Warfield, S.K., Mangin, J.F., Hüppi, P.S.: Primary cortical folding in the human newborn: an early marker of later functional development.. Brain 131(8), 2028-2041 (2008)

14. King, R.D., Brown, B., Hwang, M., Jeon, T., George, A.T.: Fractal dimension analysis of the cortical ribbon in mild Alzheimer's disease. NeuroImage 53(2), 471-479 (2010)

15. Mueller, S.G., Weiner, M.W., Thal, L.J., Petersen, R.C., Jack, C., Jagust, W., Trojanowski, J.Q., Toga, A.W., Beckett, L.: The Alzheimer's disease neuroimaging initiative. Neuroimaging Clinics of North America 15(4), 869-877 (2005)

16. Jack, C.R., Bernstein, M.A., Fox, N.C., Thompson, P., Alexander, G., Harvey, D., Borowski, B., Britson, P.J., Whitwell, J.L., Ward, C., Dale, A.M., Felmlee, J.P., Gunter, J.L., Hill, D.L.G., Killiany, R., Schuff, N., Fox-Bosetti, S., Lin, C., Studholme, C., DeCarli, C.S., Krueger, G., Ward, H.A., Metzger, G.J., Scott, K.T., Mallozzi, R., Blezek, D., Levy, J., Debbins, J.P., Fleisher, A.S., Albert, M., Green, R., Bartzokis, G., Glover, G., Mugler, J., Weiner, M.W.: The Alzheimer's Disease Neuroimaging Initiative (ADNI): MRI methods. JMRI 27(4), 685-691 (2008)

17. Freeborough, P.A., Fox, N.C., Kitney, R.I.: Interactive algorithms for the segmentation and quantitation of 3-D MRI brain scans. Computer Methods and Programs in Biomedicine 53(1), 15-25 (1997)

18. Cardoso, M.J., Clarkson, M.J., Ridgway, G.R., Modat, M., Fox, N.C., Ourselin, S.: LoAd: a locally adaptive cortical segmentation algorithm. NeuroImage 56(3), 1386-1397 (2011)

19. Hammers, A., Chen, C.H., Lemieux, L., Allom, R., Vossos, S., Free, S.L., Myers, R., Brooks, D.J., Duncan, J.S., Koepp, M.J.: Statistical neuroanatomy of the human inferior frontal gyrus and probabilistic atlas in a standard stereotaxic space. Human Brain Mapping 28(1), 34-48 (2007)

20. Warfield, S., Zou, K., Wells, W.: Simultaneous truth and performance level estimation (staple): an algorithm for the validation of image segmentation. IEEE Trans. Med. Im. 23(7), 903-921 (2004)

21. Cardoso, M.J., Modat, M., Ourselin, S., Keihaninejad, S., Cash, D.: Multi-STEPS: Multi-label similarity and truth estimation for propagated segmentations. In: 2012 IEEE Workshop on Mathematical Methods in Biomedical Image Analysis, pp. 153-158. IEEE (2012)

22. Koenderink, J.J., van Doorn, A.J.: Surface shape and curvature scales. Image and Vision Computing 10(8), 557-564 (1992)

23. Botev, Z.I., Grotowski, J.F., Kroese, D.P.: Kernel density estimation via diffusion. The Annals of Statistics 38(5), 2916-2957 (2010)

24. Nichols, T.E., Holmes, A.P.: Nonparametric permutation tests for functional neuroimaging: a primer with examples. Human Brain Mapping 15(1), 1-25 (2002) 